\title{
Eracle, Melission e il carro: un'iscrizione dal santuario dell'eroe a Tebe
}

Héraclès, Mélission et le char : une inscription du sanctuaire du héros à Thèbes

\section{Alessandra Inglese}

\section{(2) OpenEdition}

1 Journals

Edizione digitale

URL: https://journals.openedition.org/gaia/371

DOI: 10.4000/gaia.371

ISSN: 2275-4776

\section{Editore}

UGA Éditions/Université Grenoble Alpes

\section{Edizione cartacea}

ISBN: 978-2-37747-057-0

ISSN: $1287-3349$

Notizia bibliografica digitale

Alessandra Inglese, «Eracle, Melission e il carro: un'iscrizione dal santuario dell'eroe a Tebe», Gaia [Online], 21 | 2018, online dal 01 novembre 2018, consultato il 10 décembre 2021. URL: http:// journals.openedition.org/gaia/371 ; DOl: https://doi.org/10.4000/gaia.371

Questo documento è stato generato automaticamente il 10 décembre 2021.

Gaia. Revue interdisciplinaire sur la Grèce archaïque 


\section{Eracle, Melission e il carro: un'iscrizione dal santuario dell'eroe a Tebe}

Héraclès, Mélission et le char : une inscription du sanctuaire du héros à Thèbes

Alessandra Inglese

1 Fra i ritrovamenti epigrafici nel santuario di Eracle a Tebe ${ }^{1}$, insieme a vari frammenti ceramici con dediche all'eroe, sono emersi anche alcuni oggetti in bronzo: particolarmente interessante si rivela la miniatura di un finimento di carro ${ }^{2}$, sulle cui facce è incisa la medesima iscrizione; essa è composta da due parole collocate su due linee, con inversione dei termini nei due lati e con layout differente.

2 La lettura proposta dall'editore è la seguente:

lato A (fig. 1): АРГІО[.]

ME $\Lambda I \Sigma \Sigma I O N I$

lato B (fig. 2): $M E \Lambda I \Sigma \Sigma I$

ONI АРГIOI E 
Fig. 1. - Iscrizione sulla miniatura, lato A (da V. Aravantinos, Inscriptions from the Sanctuary of Herakles at Thebes, p. 197)

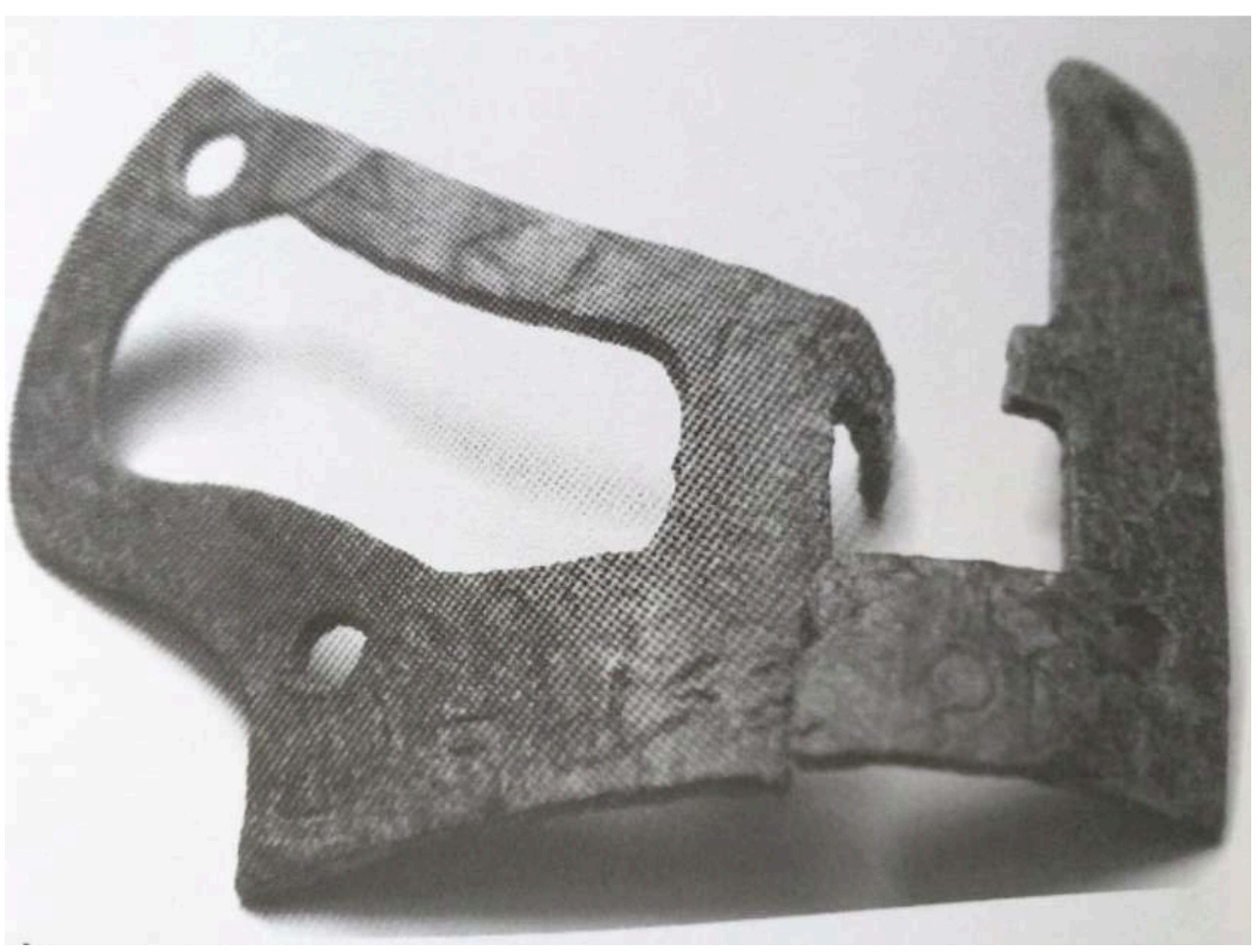

Fig. 2. - Iscrizione sulla miniatura, lato B (da V. Aravantinos, Inscriptions from the Sanctuary of Herakles at Thebes, p. 197)

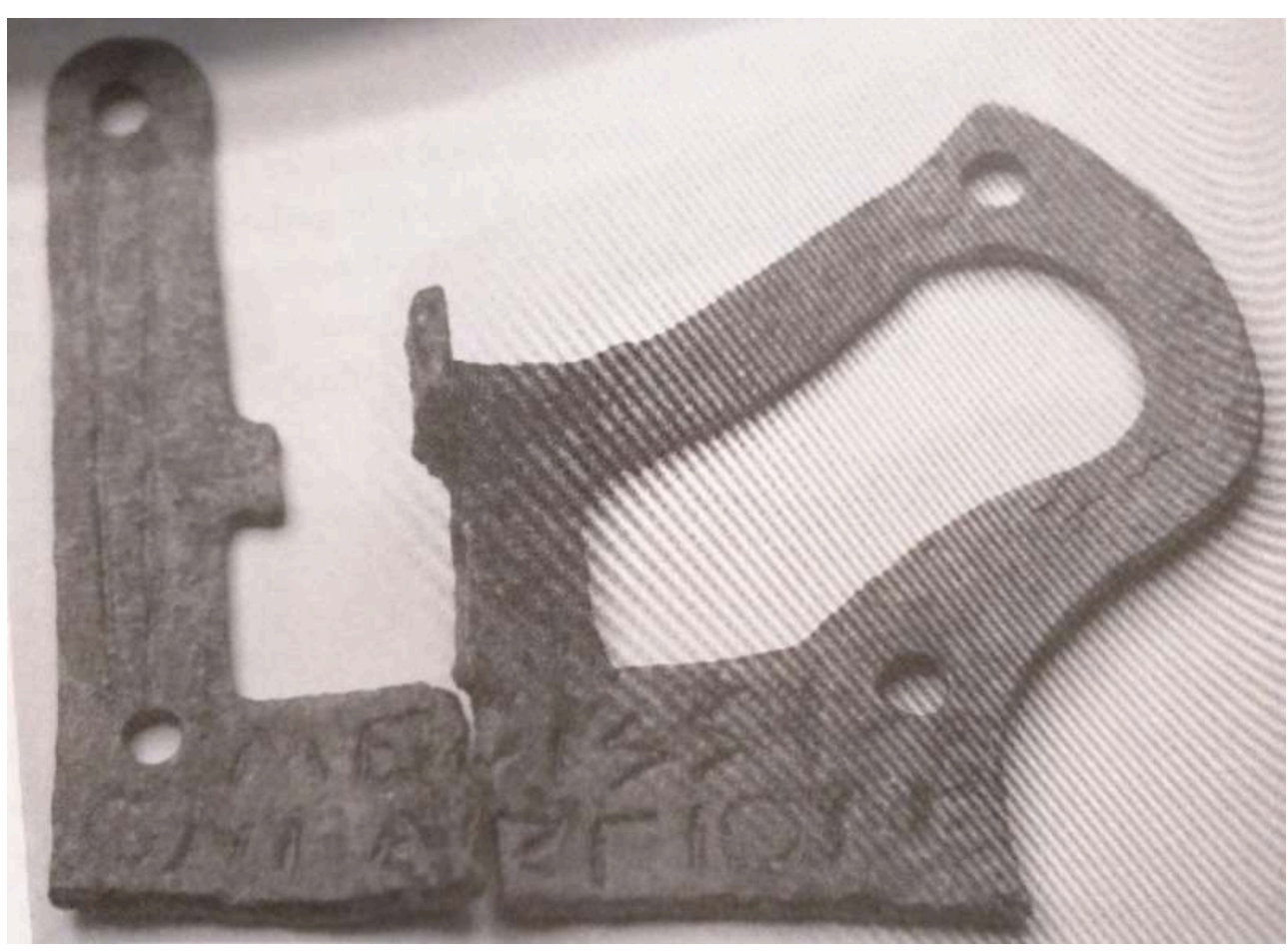

Vale subito la pena di notare che questo dono votivo iscritto sembra privo di confronti.

4 Si tratta della rappresentazione miniaturistica ${ }^{3}$ della fiancata della cassa, o predella, di un carro, probabilmente a tiro unico o al più doppio. Non siamo in grado di ricostruire 
la storia del manufatto: non sappiamo, cioè, se il pezzo fosse stato ideato volontariamente svincolato da una miniatura di carro, se si fosse trattato di un frammento residuale di bottega o se fosse un componente già in possesso del donatore; d'altra parte, nel contesto di scavo, non è stato rinvenuto il resto del veicolo e, così, allo stato attuale, l'oggetto va considerato una pars pro toto, come frequentemente avviene per altre consacrazioni nei santuari ${ }^{4}$.

5 Non sappiamo neanche quando sia stata apposta l'iscrizione, tuttavia la paleografia suggerisce una cronologia entro la prima metà del V sec. a.C., in coerenza con la datazione della miniatura proposta da Aravantinos.

6 Fra le numerose questioni sollecitate dal manufatto, vi sono la difficoltà di spiegare l'utilizzo dei quattro fori, nonchè il motivo della presenza dell'iscrizione su ambedue i lati: il finimento non poteva essere stato inchiodato, o altrimenti fissato, ad una superficie tramite i quattro fori, in quanto, oltre ad esservi l'impedimento della curvatura (cfr. lato A), non sarebbe stato possibile leggere una delle due iscrizioni. Tale difficoltà, del resto, vale anche se si suppone che l'oggetto fosse stato fissato ad una superficie curvilinea. L'editore suggerisce, dunque, che i fori servissero "for the suspension of the object by means of strings or for the attachment of other parts of the chariot $»^{5}$; quanto alla presenza della doppia iscrizione, Aravantinos ${ }^{6}$ ipotizza che avesse lo scopo di rendere il testo fruibile da ambedue i lati ${ }^{7}$.

7 Un ulteriore elemento di interesse consiste nella diversa impaginazione del testo sui due lati, con l'inversione dei termini nelle iscrizioni.

8 Su tali aspetti (doppia iscrizione, diversa impaginazione e inversione dei termini) si possono formulare le seguenti osservazioni. È possibile, innanzi tutto, rilevare che sul lato A la parola АРГІО[.] è incisa sulla stringa superiore, mentre il secondo termine, ME $\Lambda I \Sigma \Sigma I O N I$, si dispiega lungo tutta la parte inferiore, più ampia, base dell'oggetto, ed è ben inserita al di sotto dei due fori laterali.

Inoltre, nel lato $\mathrm{A}$, come rileva anche Aravantinos, le lettere ${ }^{8}$ della prima parola sono "rather large in relation to the size of the object»; i terminali infatti toccano $\mathrm{i}$ bordi superiore e inferiore della stringa. Dopo le tre lettere iniziali si notano tracce della quarta e quinta lettera, quest'ultima incisa a partire dal tratto di raccordo che congiunge la stringa superiore con il terminale curvo esterno del finimento, attualmente spezzato. Nel lato $\mathrm{B}$, invece, l'impaginato è più accurato: il modulo delle lettere è più regolare ed ambedue le parole sono collocate su due linee nel medesimo campo scrittorio, ricavato nella più ampia base inferiore, ma, come si è precedentemente accennato, in posizione invertita; inoltre, la prima parola ME $\Lambda I \Sigma \Sigma I O N I$, iscritta nello spazio entro i due fori, va a capo in corrispondenza del secondo; un poco percettibile segno divisorio la separa dal termine seguente. È possibile comunque rilevare che si riconosce un'unica mano nelle incisioni sui due lati.

10 Che vi sia una qualche intenzionalità, dunque, nell'impaginazione del lato $\mathrm{B}$ e che esso sia stato inciso dopo il lato A sembra essere suggerito, oltre che dalla maggiore accuratezza dell'incisione, anche dal fatto che è proprio nel lato $B$ che le parole assumono la posizione diversa: se infatti l'incisore avesse voluto mantenere la sequenza delle parole presenti nel lato $\mathrm{A}$, avrebbe potuto evitare l'inversione dei termini, perchè il numero delle lettere della parola qui in seconda posizione poteva rientrare perfettamente nello spazio fra i due fori e fare anche evitare l'accapo. 
11 Si può così ipotizzare verosimilmente una riscrittura finalizzata ad un miglioramento dell'aspetto grafico, attuato dall'incisore attraverso un ripensamento del layout: sembrerebbe, infatti, che nell'incidere il lato $B$, lo scrivente si sia giovato dell'esperienza - forse malriuscita - dell'impaginato del lato A, tanto da decidere per un'organizzazione diversa - e più accurata - dello spazio scrittorio.

In ogni caso, il vocabolo ME $\Lambda I \Sigma \Sigma I O N I$, al dativo, rimane collocato su entrambi i lati nella base inferiore dell'oggetto, quella di maggiore ampiezza e, quindi, la più immediatamente percepibile, verosimilmente per ricavarne un effetto enfatico.

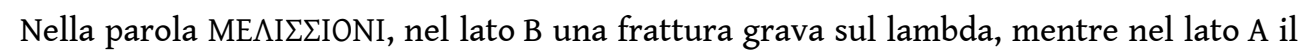
segno della medesima frattura lambisce il secondo sigma, senza inficiarne, tuttavia, la lettura.

14 In entrambi i lati si leggono, profondamente incise, le prime cinque lettere dell'altro termine АРГІО. Nel lato B, secondo la lettura di Aravantinos, questa sequenza è seguita da uno iota, con un tratto, però, non perfettamente verticale, non netto e sicuro come negli altri iota dell'iscrizione. La lettura proposta dallo studioso è, dunque, АРГІОI ${ }^{10}$.

Prima di entrare nel merito dell'interpretazione del testo, vale la pena di soffermarsi ancora un poco sulla tipologia dell'oggetto, per valutarne la congruenza con il santuario dell'eroe.

16 Una chiara traccia del legame fra Eracle e il carro è data non solo dalle fonti che raccontano la sua paideia ${ }^{11}$ e talune vicende della sua vita, ma anche dalle numerosissime immagini vascolari dell'eroe sul carro, solo o accompagnato dal compagno Iolao, ben noto auriga, o da Atena e altre divinità, nelle scene che rappresentano la sua eroizzazione.

17 Nella terza Olimpica, composta per la vittoria di Theron di Agrigento nel carro (nel 476 a.C.), Pindaro rievoca la fondazione dei giochi olimpici da parte di Eracle (vv. 13 sgg.) ed in particolare il momento in cui egli avrebbe portato nella spoglia olimpia l'oleastro sacro dal paese degli Iperborei (vv. 30-35):

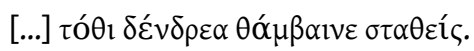

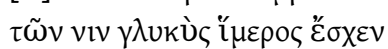

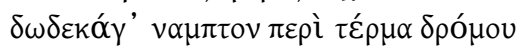

i $\pi \pi \omega v \varphi v \tau \varepsilon \tilde{v} \sigma \alpha \mathrm{l}$

[...] stupì alla vista degli alberi e ristette.

Di essi un dolce desiderio lo prese: piantarli intorno alla meta dei dodici giri nella corsa ai cavalli ${ }^{12}$;

dopo che salì all'Olimpo, Eracle affida ai Dioscuri «il mirabile agone del valore degli uomini e della guida del carro veloce».

Coerentemente con questo racconto, leggiamo in Teocrito (Idillio XXIV, 121 sgg.) un' ulteriore precisazione:

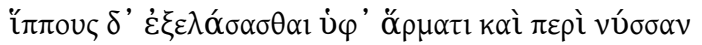

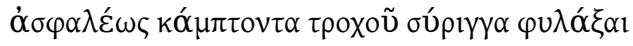

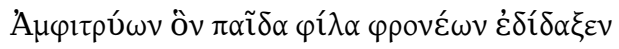

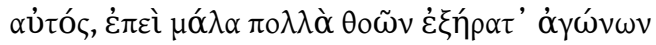

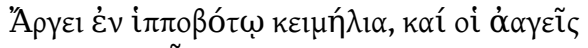

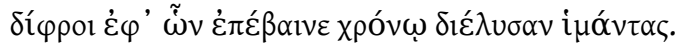

lo stesso Anfitrione insegnò al figlio ad eccitare i cavalli soggiogati dal cocchio e schivare la meta nel giro e incolume a conservare l'asse nelle ruote, 
infatti preziosi cimeli riportava dai concitati agoni ad Argo nutrice di cavalli, e i cocchi sui quali saliva, col tempo, consumavano le briglie. consacrato ad Eracle. Come ammette lo stesso Aravantinos, questa interpretazione in chiave antroponimica "runs into some difficulties if we assume that the first inscription should be read from the upper side of the siding downwards [...] i.e. APГIOI ME $\Lambda$ I $\Sigma I O N I »$, sebbene, nota lo studioso, «it has to be said that the incision of inscriptions on small object is often haphazard» ${ }^{21}$. 

a Melission, non sarebbe da connettere al mero significato dell'etnico (che, come si è detto, non è facilmente spiegabile nel contesto di un santuario tebano), quanto piuttosto alla sua connotazione di luminoso, splendente. La dedica, allora, potrebbe essere così interpretata: al luminoso Melission.

A proposito di questo termine, un'ulteriore considerazione può essere proposta. Secondo Arriano a Tiro sorgeva il più antico tempio di Eracle a memoria d'uomo, «ma non dell'Eracle $\dot{\alpha} \rho \gamma \varepsilon \tilde{o} о \varsigma$, figlio di Alcmena» ${ }^{28}$. Subito dopo lo storico parla di Cadmo, della sua presenza a Tebe e dei suoi discendenti per spiegare l'orizzonte genealogico di Eracle (la quinta generazione dopo Cadmo) ${ }^{29}$; in tale contesto egli dice che il culto di Eracle a Tiro era anteriore a quello di Eracle $\dot{\alpha} \rho \gamma \varepsilon \tau$ oc, risalente probabilmente al tempo di Edipo ${ }^{30}$. 


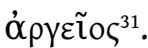
Argeios. (Aрүгі̃ov)». familiare.

Infine, ricordando l'impresa di Eracle contro Gerione, lo definisce ancora una volta

È evidente che la connotazione di Eracle come Argeios risulta insistente ed espressa con una formulazione che richiama un epiteto e che nell'excursus sia sottesa una sintesi della figura dell'eroe che si muove fra una dimensione testuale tebana e una propria di

Tale oscillazione, del resto, è presente anche in Plutarco ${ }^{32}$, il quale sostiene che «tra i poeti antichi [...] nessuno ha parlato di un Eracle fenicio o egizio, ma tutti conoscono un

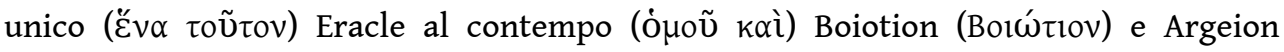

L'ascendenza argiva dell'eroe è naturalmente ricondotta alle figure di Anfitrione e Alcmena, ma M. Piérart sottolinea giustamente che «Héraclès est personellement absent des récit que les Argiens ont attachès à leur monuments» ${ }^{33}$.

Come ha notato P. Angeli Bernardini ${ }^{34}$, il carattere panellenico di Eracle - evidente nelle fonti più antiche -, viene meno col progressivo definirsi della polis, a favore degli aspetti tipicamente locali. L'eroe, benchè continui a muoversi in una prospettiva geografica ampia (specie in relazione alle vicende 'canoniche' che lo riguardano), tuttavia, a partire dalla tarda età arcaica, oscilla prevalentemente fra una fisionomia tebana ed una argiva. In particolare, secondo l'analisi della studiosa, la prima parte della sua vita risulterebbe appannaggio esclusivo di Tebe, mentre la seconda fase, quella matura, dell'età adulta, è strettamente legata all'Argolide (soprattutto a causa alle fatiche impostegli da Euristeo).

Sulla scorta 1) del passo plutarcheo, che rende per così dire 'consustanziale' la natura di argeios e quella tebana di Eracle, 2) dell'indicazione di Piérart, che mette in guardia dall'attribuire ad Eracle il significato di $\dot{\alpha} \rho \gamma \varepsilon \tilde{o} о \varsigma$ nel mero senso dell'etnico, 3) delle indicazioni linguistiche di Chantraine, e 4) sulla base delle considerazioni relative allo

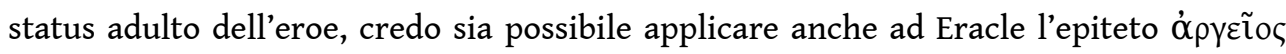
nell'accezione di luminoso, brillante. In altre parole, il termine argeios gioca intenzionalmente su un doppio livello semantico: da una parte si richiama la natura 'luminosa' dell'eroe, dall'altra si definisce un legame con l'orizzonte argolico; in sostanza con gli Argioi, coloro che portano la propria luminosità nel nome.

Eracle resta comunque tebano, ma è anche ó affrontato vittoriosamente nella seconda parte della sua esistenza ${ }^{35}$; quanto a Melission, anch'egli a Tebe in qualche misura partecipa della natura luminosa dell'eroe.

Non si possono ignorare in questa ricostruzione le Istmiche III e IV di Pindaro, entrambe dedicate ad un tal Melissos, vincitore per due volte nella corsa del carro, nonché membro della famiglia dei Cleonimidi, ben nota a Tebe come allevatrice di cavalli e risultata vittoriosa a più riprese ad Atene e a Sicione nelle competizioni con i carri.

Non si tratta ovviamente dello stesso personaggio dell'iscrizione, ma si può suggerire l'ipotesi secondo la quale si potrebbe trattare di un membro della medesima famiglia: come è solita fare, l'onomastica greca, attraverso l'inserzione di suffissi, costruisce varianti negli antroponimi per differenziare un individuo da altri dello stesso gruppo

5 Il collegamento fra Melissos ed Eracle nella IV Istmica è il fulcro dell'ode e, in un certo senso, il fulcro dell'eulogia familiare. 

vinto come pancraziaste (una volta come pais e due da adulto) nelle Herakleia o Iolaieia, agoni annuali di grande importanza che si tenevano, a detta del poeta, presso le porte Elettre. Al vincitore andava in premio una corona di mirto (le feste erano in onore dei

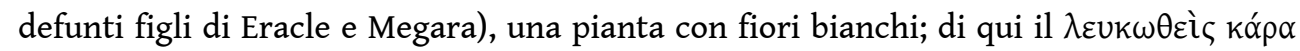
uúptoıc (Istmica IV, 69), il capo imbiancato ${ }^{36}$ dal mirto o, come traduce Privitera ${ }^{37}$, il capo luminoso di mirto di Melissos. anche essere considerato un antroponimo, è formulabile un'ipotesi ulteriore: la dedica sul finimento potrebbe essere stata indirizzata ad un personaggio eroizzato, membro illustre di un genos evidentemente ben noto a Tebe dal momento che non si ritiene necessario indicarlo con maggiore precisione.

Melission sarebbe, così, una figura percepita come esemplare, propria di una cultura familiare che nella prima metà del V sec. a.C. tesse su più livelli, e con diversi strumenti, la trama della propria autorappresentazione, collegandola a più riprese $\mathrm{e}$ in varie forme ad Eracle.

Nella dimensione votiva, in relazione cioè ad un momento 'materiale', è stato scelto un oggetto che allude all'eroe tebano e al contempo risulta connotante la committenza; sul manufatto viene aggiunta un'iscrizione che chiarisce ulteriormente il messaggio alluso dal solo dono votivo: il destinatario della dedica, un eroe familiare, condivide con l'eroe della città la natura argeia.

A questo momento per così dire visuale, negli stessi anni, viene affiancata la dimensione poetica ${ }^{38}$ : la vittoria nella corsa dei carri nelle Istmiche di un membro della famiglia avrà fornito un'ulteriore occasione alla strategia propagandistica familiare; è così commissionato un epinicio ad un concittadino illustre che fa risuonare chiaramente il kleos del genos, scegliendo di insistere su una precisa dimensione eroica e rendendo eterno quanto il bronzo il rapporto con Eracle.

\section{BIBLIOGRAFIA}

ANGELI BERNARDINI Paola, Eracle: una biografia eroica tra epos arcaico, poesia lirica e tradizioni locali, in E. Cingano (ed.), Tra panellenismo e tradizioni locali: generi poetici e storiografia, Alessandria, Dell'Orso, 2010.

ARAVANTINOS Vasileios, The Inscriptions from the Sanctuary of Herakles at Thebes:an Overview, in N. Papazarkadas (ed.), The Epigraphy and History of Boeotia, Leiden, Brill, 2014.

BINTLIFF John \& SNODGRASS Antony, The Cambridge/Bradford Boeotian Expedition: The First Four Year, «Journal of Field Archaeology», XII, 1985, pp. 123-161.

BONANNO Maria Grazia, L'allusione necessaria. Ricerche intertestuali sulla poesia greca e latina, Roma, Ateneo («Filologia e critica», 63), 1990. 
BREMMER Jan, Mythe et rituel dans l'initiation d'Héraclès, in C. Calame \& P. Ellinger (edd.), Du récit au rituel par la forme esthétique. Poèmes, images et pragmatique cultuelle en Grèce ancienne, Paris, Belles Lettres, 2017.

BRILLANTE Carlo, La paideia di Eracle, in C. Bonnet \& C. Jourdain-Annequin (edd.), Héraclès. D’une rive à l'autre de la Méditerranée. Bilan et perspectives (Actes de la table ronde, Academia Belgica École française de Rome, 15-16 septembre 1989), Bruxelles-Rome, Institut historique belge de Rome («Études de philologie, d'archéologie et d'histoire anciennes », 28), 1992, pp. 199-222.

CHANTRAINE Pierre, Dictionnaire étymologique de la langue grecque. Histoire des mots, Paris, Kincksieck, $1999^{2}$.

FURTWÄNGLER Adolf, Die Bronzen von Olympia (Olympia IV), Berlin, Asher, 1890.

GOW Andrew Sydenham Farrar, Theocritus, I-II, Cambridge, Cambridge University Press, $1952^{2}$.

GENTILI Bruno, CATENACCI Carmine, GIANNINI Pietro \& LOMIENTO Liana, Pindaro. Le Olimpiche, Milano, ed. Valla, Mondadori, 2013.

MOGGI Mauro \& OSANNA Massimo, Pausania. Guida della Grecia, Libro IX: La Beozia, Milano, ed. Valla, Mondadori, 2010.

PIÉRART Marcel, Les honneurs de Persée et d'Héraclès, in C. Bonnet \& C. Jourdain-Annequin (edd.), Héraclès. D'une rive à l'autre de la Méditerranée. Bilan et perspectives (Actes de la table ronde, Academia Belgica - École française de Rome, 15-16 septembre 1989), Bruxelles-Rome, Institut historique belge de Rome («Études de philologie, d'archéologie et d'histoire anciennes », 28), 1992, pp. 223-244.

PILZ Oliver, The Uses of Small Things and the Semiotics of Greek Miniature Objects, «Pallas», 86, 2011, pp. 15-30.

PRIVITERA Giuseppe Aurelio, Pindaro. Le Istmiche, Milano, ed. Valla, Mondadori, 2014.

RAGONE Giuseppe, Riflessioni sulla documentazione storica su Fidone di Argo, in C. Bearzot \& F. Landucci (edd.), Argo, una democrazia diversa, Milano, Vita e pensiero, 2006.

SCHACHTER Albert, Cults of Boiotia, IV, London, University of London, Institute of Classical Studies, 1986.

\section{NOTE}

1. Aravantinos $(2014,149-210)$.

2. H. max. cm. 8,6, l. max. cm. 8,8.

3. Vale la pena di ricordare la riflessione, con approccio semiotico, da parte di Pilz (2011, 15-30), sul significato degli oggetti miniaturistici come offerte nei santuari.

4. Per rimanere nello stesso ambito, a tale categoria di oggetti votivi appartengono, per esempio, le dediche del morso del tiro del carro o il noto terminale di timone di carro a testa d'aquila rinvenuto ad Olimpia (sul quale cfr. Furtwängler, 1890, $n^{\circ} 975$, tab. LVII). Tale procedimento è attestato soprattutto per la consacrazione di singole parti della panoplia che ad essa evidentemente alludono.

5. Aravantinos (2014, 196-197).

6. Ibid.

7. Si mantiene la denominazione usata dall'editore per definire i due lati dell'oggetto: lato A (quello esterno) e lato B (quello interno). La sequenza A-B non sembra dettata dall'espressione 
del messaggio e non sembra funzionale ad una migliore comprensione del testo da parte del lettore (cfr. infra).

8. H. cm. 0,7-0,8.

9. Ibid., 197.

10. L'editore suggerisce, inoltre, con estrema cautela, che quest'ultimo iota, sempre sul lato $B$, fosse seguito da un'ulteriore lettera che richiamerebbe un epsilon con la sola parte superiore del tratto verticale ben inciso, su cui si innesterebbe un'asta orizzontale in alto, ma non nella sezione centrale. La parte finale del tratto verticale sarebbe scomparsa in un punto rovinato, ma parte di una terza asta potrebbe avere un andamento obliquo ascendente, diverso dunque da quella superiore, che si sarebbe dovuto innestare nel punto in cui il terminale inferiore del tratto verticale è rovinato; la lettura, in tal modo, risulterebbe АРГІОІЕ. Il presunto epsilon, tuttavia, non permette di comporre alcuna parola con un senso compiuto e, d'altra parte, le tre eventuali aste sembrano essere dei segni accidentali.

11. Cfr. anche Brillante (1992, 199-222).

12. La traduzione è di Gentili (2013). Su Eracle e la fondazione dei giochi olimpici in Pindaro, vedi anche Olimpica II (vv. 3 sgg.), V (vv. 5 sgg.), VI (vv. 68 sgg.), X (vv. 43 sgg.) e Nemea X (vv. 32, sgg.).

13. [Apollodoro], Biblioteca, II, 4,9. Tra le competenze che l'eroe doveva acquisire, alla guida del carro, è assegnato il primo posto; seguono la lotta (appresa da Autolico), il tiro con l'arco (da Eurito), l'uso delle armi (da Castore) e, infine, la cetra (da Lino). È stato opportunamente notato che la guida del carro «can hardly have been part of the initial education, given that one needs to have the necessary strength to steer horses. [...] Charioteering was the reverse of being treated as a young foal or colt, like the Spartan boys in the agela. To be able to drive a chariot demonstrated that one had outgrown the age of boys who still had to be domesticated» (Bremmer, 2017). Non a caso, diversamente dallo Ps. Apollodoro, che pone come primo momento della paideia di Eracle la guida del carro, in Teocrito, più coerentemente, questa abilità è l'ultima ad essere nominata.

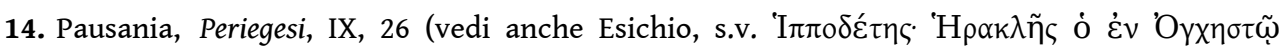

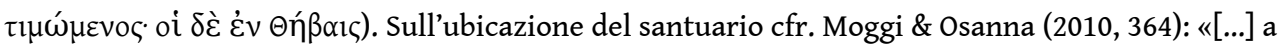
giudicare dal percorso seguito da Pausania doveva trovarsi all'estremità della piana tebana, una zona di confine»; vedi anche Schachter (1986, 12-13). Per un'ubicazione presso Onchesto vedi invece Bintliff \& Snodgrass (1985, 139-140).

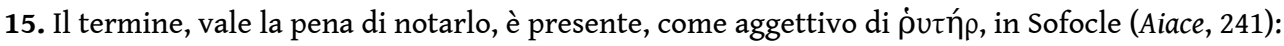
il riferimento è proprio alla briglia o ad una correggia dei finimenti.

16. Su cui Ragone (2006, 47 sgg.).

17. Va a questo proposito evidenziato, infatti, che nel santuario non sono presenti iscrizioni per altre divinità, tranne due epigrafi per Apollo Ismenio, che è comunque fra le divinità principali di Tebe (un'iscrizione sul bordo di un kantharos in bronzo e un'altra frammentaria su pietra).

18. Aravantinos $(2014,199)$.

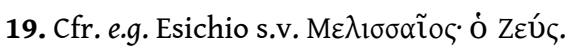

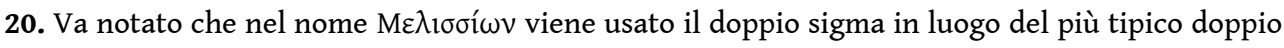
tau; le occorrenze epigrafiche delle due forme, tuttavia, anche nelle varianti onomastiche in Beozia datano per lo più a partire dal IV sec. a.C. (cfr. LGPN IIIB, si registra un Mć $\lambda_{\imath} \tau \tau \alpha$ presso Kopai, un unico caso di $\mathrm{V}$ sec.) e non permettono di considerare eccentrica la variante con doppio sigma nell'età qui interessata.

21. Aravantinos $(2014,198$, n. 82).

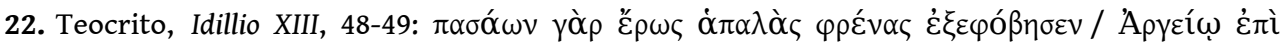
$\pi \alpha 1 \delta i ́$.

23. Gow (1952² I, 98 e II, 241).

24. Bonanno (1990, 203-206, la citazione è a p. 204).

25. Ibid. 
26. L'editore Latte non accetta il riferimento ad Esiodo, al contrario Schmidt accetta il manoscritto Marciano. I due studiosi, tuttavia, ammettono l'equivalenza.

27. In riferimento, per esempio, al nome della nave Argo, cioè «veloce».

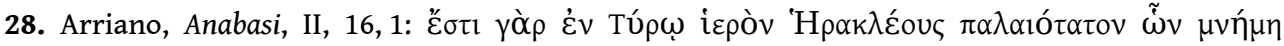

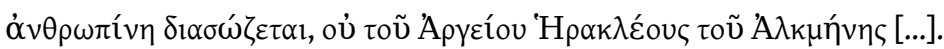

29. Per la quinta generazione dopo Cadmo, cfr. anche Erodoto II, 44,4 a proposito del santuario dedicato all'Eracle fenicio a Taso.

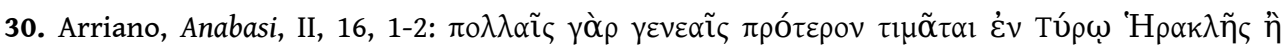

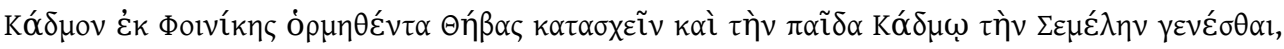

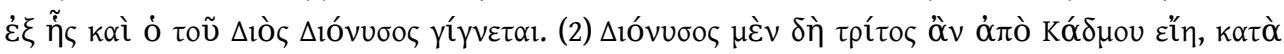

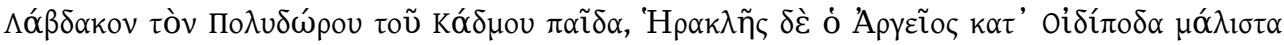

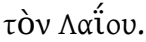

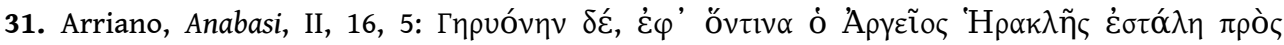

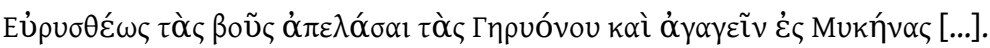

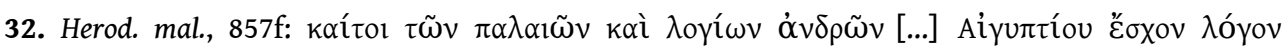

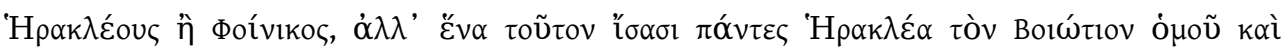
Aрүعĩov.

33. Piérart (1992, 230).

34. Angeli Bernardini $(2010,405)$.

35. L'età adulta che risulta anche coerente con l'acquisizione dell'abilità nella guida del carro, come è stato rimarcato dalle considerazioni di Bremmer.

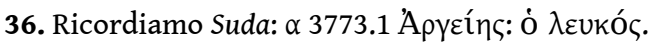

37. Privitera $(1982,69)$.

38. Sull'associazione in Pindaro tra la composizione poetica e manufatto artistico-figurativo vedi Gentili (2013, 416-417, n.3), con richiami a Pitica III (113 sgg.) e VI (7 sgg.), Nemea IV (81) e VIII (46): «Pindaro, in Nemea $V, 1$ sgg. non manca di rivendicare il primato della poesia sull'arte figurativa e in particolare sulla statuaria, medium concorrente nella celebrazione di vittorie: la parola cantata "viaggia sopra ogni nave", è superiore per mobilità e portata comunicativa».

\section{RIASSUNTI}

Fra i ritrovamenti epigrafici nel santuario di Eracle a Tebe particolarmente interessante si rivela un oggetto in bronzo iscritto: una miniatura di un finimento di carro, databile entro la prima metà del V sec. a.C. La tipologia dell'oggetto risulta coerente con la fisionomia dell'eroe, ma l'iscrizione presenta una certa complessità interpretativa, già denunciata dal primo editore V. Aravantinos. L'epigrafe è composta da due termini, in uno delle quali sembra potersi riconoscere un'accezione più estesa che connoterebbe lo stesso Eracle e che mostrerebbe un'ulteriore caratteristica della natura dell'eroe.

Parmi les découvertes épigraphiques effectuées dans le sanctuaire d'Héraclès à Thèbes, un objet en bronze portant une inscription a retenu notre attention. Il s'agit d'une miniature d'un harnachement de char, datable de la première moitié $\mathrm{du} \mathrm{v}^{\mathrm{e}}$ siècle av. J.-C. La typologie de l'objet est cohérente avec la physionomie du héros, mais l'interprétation de l'inscription se révèle complexe, comme l'a affirmé son premier éditeur V. Aravantinos. L'inscription se compose de 
deux mots: dans l'un d'eux il serait possible de reconnaître une acception plus étendue qui évoquerait Héraclès et qui mettrait en évidence l'une des caractéristiques de la nature de ce héros.

INDICE

Mots-clés : Thèbes, Héraclès, épigraphie grecque, miniature en bronze

Parole chiave : Tebe, Eracle, epigrafia greca, miniatura di bronzo

\section{AUTORE}

\section{ALESSANDRA INGLESE}

Università degli Studi di Roma «Tor Vergata».

Alessandra Inglese enseigne l'épigraphie grecque à l'université de Rome « Tor Vergata » depuis 2009. Elle est aussi responsable scientifique du projet Epigrammata : rencontre biannuelle d'épigraphie grecque. Elle est membre du comité de rédaction de la revue Rationes Rerum (rivista di Filologia e Storia). 